\title{
Maleta saludable
}

\section{Educación Física}

Adrián Costa Chacón al287043@uji.es

Tamara Sánchez Núñez

al287191@uji.es 


\section{Resumen}

Esta investigación pretende dar una opción novedosa y atractiva a docentes especialistas en la materia de Educación Física (EF), concretamente en la etapa de primaria, para esos días que, por varios motivos, no pueden disponer del espacio asignado para realizar sus sesiones. Se trata de la elaboración de un recurso didáctico, mediante el cual podamos trabajar los hábitos saludables, contenido de esta asignatura, que no se trabajan de forma específica en el curso escolar.

En la actualidad con las nuevas tecnologías, el ritmo de vida estresante y las empresas de comida precocinada o rápida, no se atiende adecuadamente la salud de nuestro organismo y es cuando surgen el sedentarismo, la obesidad infantil u otras enfermedades. Otro rasgo a destacar es que el alumnado no tiene integrado muchos de los hábitos saludables, bien por desconocimiento o por no tener la costumbre. De este modo, podremos educar a este colectivo para que implante una serie de hábitos en su día a día.

Para ello, hemos creado una maleta didáctica para trabajar contenidos del bloque 4 del currículum de EF: actividad física y salud. Así pues, con la utilización de este recurso, el alumnado aprenderá hábitos saludables de una manera lúdica y atractiva donde se sienta partícipe en su propio aprendizaje.

Palabras claves: Educación Física, hábitos saludables, recurso didáctico, primaria, docente y aprendizaje.

\section{Introducción}

En primer lugar, para confeccionar la base teórica de esta investigación, pensamos que debemos definir cada uno de los términos que utilizaremos y explicar la importancia que tienen con el fin de adecuar el conocimiento de estos y evitar confusiones. De este modo, cualquier persona que lea esta investigación podrá entender todo su contenido, sea entendida o no del tema tratado.

¿Qué es el hábito de salud? Si buscamos la definición de cada palabra en la Real Academia Española de la lengua (RAE), podemos encontrar para el término hábito varias definiciones de las cuales nos quedamos con la siguiente: «Modo especial de proceder o conducirse adquirido por repetición de actos iguales o semejantes, $u$ originado por tendencias instintivas» (RAE, 2017). Del concepto salud también encontramos varias definiciones, pero nos quedamos con la siguiente: «Estado en que el ser orgánico ejerce normalmente todas sus funciones» (RAE, 2017). Es decir, para que un organismo goce de salud debe proceder con una serie de acciones para que fluyan 
correctamente todas las funciones. Ahora bien, si juntamos los dos términos podemos decir que hábitos saludables son aquellos que un individuo debe hacer, como costumbre en su vida diaria, para que su organismo funcione correctamente.

Encontramos la definición de la Organización Mundial de la Salud (OMS) que desde su constitución define así el concepto de salud: «el estado de completo bienestar físico, mental y social, y no la mera ausencia de enfermedad" (1948). En base a esta podemos decir que la salud no solo abarca el estado del cuerpo físico del individuo y que, por lo tanto, la asignatura de EF, no solo debe tratar el cuerpo sino también las relaciones sociales y el contexto donde se practique, puesto que todo influye en la persona a la hora de la práctica de esta asignatura o en cualquier tipo de actividad física de tiempo libre, fuera del horario escolar. Todo esto lo podemos respaldar con palabras de Sánchez Bañuelos (1996: 88), que dice así:

Los objetivos de la Educación Física, hoy en día, se mueven en torno a parámetros educativos impregnados de un carácter integral y vivencial, vinculados con la formación y el desarrollo de los aspectos esenciales de la motricidad humana. Una motricidad entendida no en sí ni por sí misma, sino con una estrecha vinculación con las relaciones interpersonales sociales y ambientales de la persona.

Por otra parte, podemos encontrar muchas definiciones para la Educación para la Salud (EpS) y todas partirán de la definición de la OMS. Una de estas la podemos hallar en la Ley Orgánica General del Sistema Educativo español del 3 de octubre de 1990 (LOGSE), de la mano de la profesora Perea (1992: L), que fue la encargada de incluir el término EpS dentro de la ley educativa:

Un proceso de educación permanente que se inicia en los primeros años de la infancia orientado hacia el conocimiento de sí mismo en todas sus dimensiones tanto individuales como sociales, y también del ambiente que le rodea en su doble dimensión, ecológica y social, con objeto de poder tener una vida sana y participar en la salud colectiva.

Con esta reforma de la LOGSE es cuando se introduce la importancia de la salud en las escuelas, para así promocionar un estilo de vida saludable, concienciar a la sociedad de la necesidad de aplicar los hábitos saludables adecuados a nuestra vida diaria y combatir la obesidad infantil y otras enfermedades que preocupan en la sociedad actual. Pero, ¿qué es un estilo de vida saludable? Mendoza, Sagrera y Batista (1994: 17) definen estilo de vida «como un conjunto de patrones de conducta que caracterizan la manera general de vivir de un individuo o grupo». Más adelante sigue 
ampliando este concepto, diciendo (Mendoza, Sagrera y Batista, 1994: 17):

Dentro de una determinada sociedad no hay un único estilo de vida saludable, sino muchos, cualquiera que sea la definición de salud que se adopte. El estilo ideal de vida saludable no existe, afortunadamente, porque la que se vive es saludable. Además, cualquier estilo de vida calificado globalmente como saludable puede contener elementos no saludables, o saludables en un sentido pero no en otro.

La OMS y Mendoza et ali, entre otros, afirman que existen múltiples estilos de vida saludables, por lo que este término llega a ser bastante complejo y nos lleva a la pregunta, ¿cuál es el estilo de vida saludable adecuado? Como sabemos, cada individuo tiene unas características diferentes a otro. Hay diversos factores que nos influyen, como los biológicos (sexo, edad, genética) y los psicológicos (socioeconómicos, culturales, políticos).

A continuación exponemos una fotografía extraída de Delgado y Tercedor (2002), siguiendo a Mendoza y otros (1994), que explica visualmente este concepto, con la teoría del Modelo Ecológico de Bronfenbrenner (1979) presente:

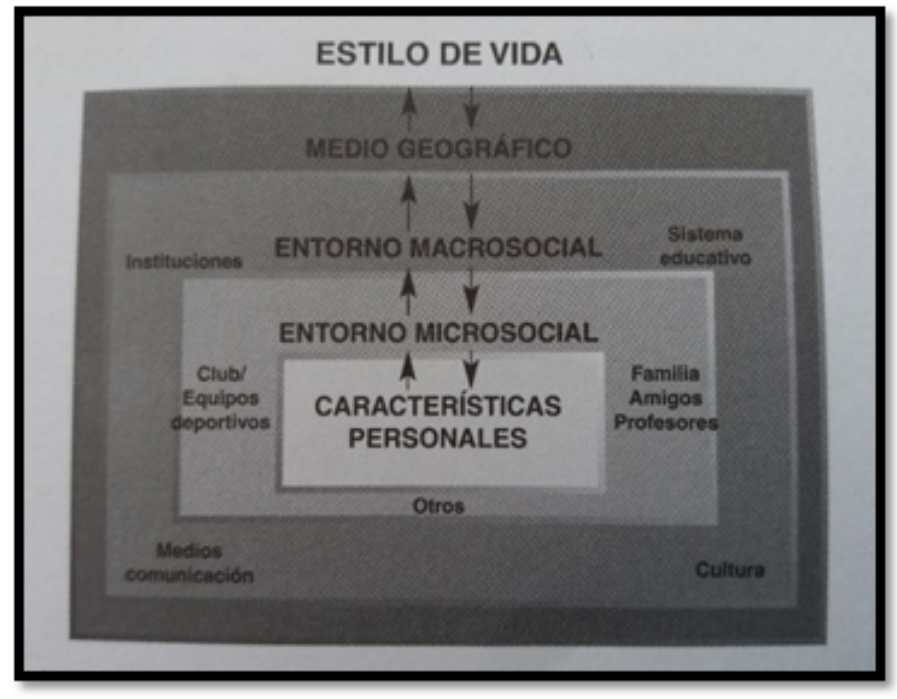

Figura 1. Factores que determinan el estilo de vida de las personas.

Delgado y Tercedor (2002: 73). Adaptado de Mendoza, Sagrera y Batista (1994)

A pesar de todos los tipos de estilos de vida saludables, Delgado y Tercedor (2002: 73) nos aclaran este concepto:

No obstante, y a pesar de esta multiplicidad de estilos de vida saludables, sí que existe un consenso avalado por la investigación científica y la riqueza cultural, mediante el cual se puede identificar 
una conducta como saludable (p.e. la práctica de actividad física moderada) o no saludable (p. e. el consumo de sustancias tóxicas).

Volviendo a la EpS, debemos saber qué aspectos tiene que tener en cuenta la escuela en la salud de los menores. Considerando su importancia en la temprana edad, queda claro que la escuela debe ser un pilar básico para su desarrollo y aprendizaje por varias razones como las que nos destacan tanto Hernán y otros (2001) como Pozuelos y Travé (1999) (citados en Delgado y Tercedor, 2002: 40):

- Porque es algo que afecta a nuestra calidad de vida (presente y futura).

- Porque es un ámbito complejo que afecta a muchos factores: sociales, ambientales, biológicos, económicos,... y por ello no es exclusivamente sanitario.

- Porque su preservación precisa de nuestro compromiso y esto se aprende: no es espontáneo, ni fortuito. Necesita de un proceso continuado y reflexivo.

- Porque la escuela sola no consigue pero su apoyo la propicia y facilita.

En la actualidad, el currículum de educación vigente, Ley Orgánica para la Mejora de la Calidad Educativa de 9 diciembre del 2013 (LOMCE, 2013: 46), nos indica:

En cuanto a la adopción de hábitos saludables es muy importante tener en cuenta que se estima que hasta un $80 \%$ de niños y niñas en edad escolar únicamente participan en actividades físicas en la escuela, tal y como recoge el informe Eurydice, de la Comisión Europea de 2013; por ello la Educación Física en las edades de escolarización debe tener una presencia importante en la jornada escolar si se quiere ayudar a paliar el sedentarismo, que es uno de los factores de riesgo identificados, que influye en algunas de las enfermedades más extendidas en la sociedad actual.

Con todo lo analizado anteriormente, y desde nuestra perspectiva sobre la asignatura de EF, pensamos que se debe potenciar una serie de hábitos saludables, de acuerdo con la definición anterior de la OMS, para que el alumnado goce de una buena salud tanto física, mental y social. Por eso, el docente de EF debe fomentar cómo realizar correctamente la actividad física atendiendo a las posturas y acciones adecuadas para evitar lesiones o enfermedades, así como la atención a la higiene personal, realizar programas con colaboración del centro y familiares, para fomentar una alimentación sana y equilibrada, además de potenciar el descanso y la relajación y respiración. Desde nuestro punto de vista, estos motivos son los que hacen que sea más importante tratar y concienciar sobre estos aspectos en la Didáctica de la Educación 
Física, englobada dentro del bloque de contenido actividad física y salud.

Asimismo, consolidar los hábitos de salud en niños de la etapa educativa de primaria no es tarea fácil para el docente de Educación Física, puesto que, como afirman Kelder y otros (1994), los niños no llegan a fijar estos hábitos en su vida diaria hasta los 11 años de edad. Sin embargo, estos contenidos tienen que trabajarse durante toda la etapa para llegar a asimilarlos completamente y poder involucrarlos en el resto de la vida adulta del individuo.

Para finalizar con los hábitos saludables, creemos necesario citar las palabras de Sánchez Bañuelos (1996: 261), que nos indica la importancia de estos hábitos en las clases de EF:

No obstante, tal como se ha visto, es muy relevante para la salud presente y futura del individuo, el que ya desde la etapa escolar se adopten hábitos de adherencia a la práctica del ejercicio físico. La educación física no puede estar al margen, ni mucho menos constituir un elemento negativo para que estos hábitos se adquieran, ya que si así fuera dicha materia perdería gran parte de su sentido en el curriculum escolar.

\section{Objetivos}

El propósito de nuestra investigación es diseñar un recurso didáctico alternativo y dinámico para el docente de EF, cuando este no pueda utilizar el espacio asignado, ya sea por motivos meteorológicos, de ocupación, etc.

Relacionando el currículum con este proyecto, que está centrado en el bloque 4 de contenidos del currículum de Educación Primaria, titulado "Actividad física y salud", podemos extraer un objetivo más específico: conseguir, mediante este recurso, que el alumnado reconozca y adquiera los hábitos saludables a través de actividades individuales y colectivas, respetando las normas y a los demás.

\section{Material y métodos}

En este apartado procedemos a explicar en qué consiste el recurso que hemos diseñado, así como las instrucciones para poder utilizarlo en el aula con el alumnado en esos días que no se pueda realizar las clases de EF en el patio o gimnasio. Este proyecto consiste en la elaboración de una maleta didáctica.

En nuestro caso, la maleta didáctica se compone de varios juegos de mesa donde se tratan los hábitos saludables. Esperamos que, por medio de este recurso, el alumnado que lo ponga en práctica adquiera nuevos conocimientos sobre este tema y refuerce los que ya dispone. Este recurso, además de utilizarlo en los días donde no se pueda salir al patio para llevar a cabo la sesión, también 
es un buen recurso para tratar el tema de los hábitos saludables, que se suele tratar de manera transversal en diferentes asignaturas, entre ellas la de EF. Con esto no queremos decir que se tenga que usar solo en los días que no podemos utilizar el patio del colegio, sino que podemos usarlo siempre que creamos conveniente para trabajar los hábitos saludables de manera más específica.

Nosotros hemos elaborado este recurso con un nivel educativo dirigido concretamente a alumnado de 5. y 6.ㅇ de primaria. No obstante, estos juegos se pueden adaptar a cada nivel educativo modificando las preguntas, pruebas y reglas, según el contexto de aula o nivel madurativo del alumnado.

Ahora pasamos a explicar detalladamente cada juego que hemos diseñado para nuestra maleta didáctica:

\section{Juegos de la maleta saludable}

\section{Trivisaludable:}

El juego consta de un tablero en el que mover las fichas, varias tarjetas con preguntas y respuestas de las diferentes temáticas de los hábitos saludables, fichas de diferentes colores, un dado, un reloj (de arena, digital o analógico) y pirámides de colores, formadas por piezas de juguetes montadas en forma de pirámide (también se puede emplear otro material si se cree conveniente).

Este juego consiste en ir pasando por las diferentes casillas de colores acertando las preguntas y conseguir las pirámides saludables. Para conseguirlas es necesario estar en las casillas grandes y responder bien a la pregunta correspondiente. Hay cinco casillas grandes en forma de pentágono de diferentes colores. Una vez obtenidas las cinco pirámides, se deberá volver a la casilla del centro para ganar la partida.

Reglas:

- Para empezar, el jugador o grupo que obtenga el mayor número al lanzar el dado será el que empiece la partida. En caso de empate los implicados seguirán lanzando hasta que haya un vencedor.

- El orden de lanzamiento será el de las agujas del reloj.

- Todos los jugadores empiezan desde la casilla del centro.

- Cada jugador podrá lanzar el dado un máximo de tres veces seguidas si acierta las preguntas.

- Las preguntas las formula el jugador o grupo situado a su derecha.

- El tiempo máximo para responder a las preguntas, después de que otro jugador la haya leído, será de 30 segundos.

- En caso de no acertar la pregunta o sobrepasar el tiempo, pasará el turno al siguiente jugador o grupo. 
- Para conseguir pirámide, la ficha debe estar en alguno de los pentágonos grandes antes indicados y se debe acertar la pregunta.

- Se pueden desplazar las fichas en la dirección que el jugador quiera con el número que indique el dado.

- Máximo cinco jugadores, si se juega individualmente, o cinco equipos, si se juega de manera grupal.

\section{Corazón saludable:}

Este juego se compone de un tablero con diferentes casillas de colores que van en una sola dirección, fichas para cada jugador o equipo, un dado, tarjetas de preguntas y pruebas, bloc de folios en blanco y lápices.

El juego consiste en ir pasando por las diferentes casillas y superar las diferentes pruebas, ya sean de preguntas o dibujo. Los jugadores empezarán desde la casilla de salida y seguirán la dirección del tablero hasta llegar a la última casilla de meta, donde estará el corazón para ganar. Cada vez que un jugador tire el dado avanzará según las casillas que el número del dado indique y otro jugador extraerá la tarjeta correspondiente de la temática de la casilla y le dirá en qué consiste la prueba. También habrá casillas con dibujos o indicaciones donde se deberá realizar la acción que se diga, ya sea a favor o en contra del jugador que caiga en esta casilla.

\section{Reglas:}

- Si un jugador cae en la casilla donde hay un signo de un lápiz tendrá que dibujar lo que esté escrito en la tarjeta. Si se juega individualmente, será el jugador que le toque el próximo turno el que tenga que adivinar de qué trata el dibujo. Si se juega por grupos, habrá un encargado de dibujar y el resto de su mismo equipo deberá adivinarlo.

- En las casillas con un signo de pregunta, se realizarán preguntas con y sin opciones en las que el jugador deberá acertar la respuesta para superar la prueba.

- Si el jugador responde correctamente, continúa jugando, hasta un máximo de tres veces seguidas. Si no supera la prueba, pasa el turno.

- Empieza el juego quien más puntuación haya conseguido al lanzar el dado.

- Cada prueba se tiene que llevar a cabo y superar en un máximo de un minuto; pasado el tiempo, pasa el turno.

- Gana el jugador que llegue primero a la casilla de meta.

\section{Vocabulario saludable:}

Este juego sirve para aprender y ampliar vocabulario jugando con los compañeros. Consiste en buscar palabras de los ítems 
indicados que empiecen con la letra elegida a suertes, con una ruleta. Quien más palabras tenga correctas, tendrá más puntos. Los materiales necesarios serán una ruleta con el abecedario; cuadernos con las tablas de los ítems, uno para cada jugador; lápices y gomas, según los jugadores que sean, y un reloj (de arena, digital o analógico). Los diferentes ítems están relacionados con la alimentación.

Reglas:

- Para elegir la letra con la que se juegue rodará la ruleta cada vez un jugador. La letra que indique la flecha de la ruleta será con la que jugarán esa ronda. Se jugará en el sentido de las agujas del reloj.

- Se acaba la ronda cuando pase una de las dos cosas siguientes: cuando se acabe el tiempo, un máximo de 2 minutos, o cuando un jugador diga «iHECHO!», momento en el que todos levantan los lápices.

- Cuando los jugadores levanten los lápices, se empieza a comprobar si las palabras son correctas. Si es por tiempo, empieza a comprobarse la tabla del jugador que haya rodado la ruleta, y si es por el jugador que ha acabado antes, se empieza a comprobar por este y después se sigue según en el sentido de las agujas del reloj.

- Si un jugador tiene todas las palabras completas y correctas obtiene 10 puntos, si le falta alguna palabra o hay alguna que no es correcta, se descuentan 2 puntos por palabra. Al final del juego se suman los puntos de cada ronda y gana el que más puntos haya obtenido.

- No hay máximo de jugadores.

\section{Fichas saludables:}

Se trata del juego clásico del dominó, pero en vez de haber puntos que representan números, las fichas tendrán dibujos de hábitos correctos e incorrectos. De este modo, el alumnado observará los dibujos de las fichas mientras juega y podrá diferenciar los hábitos correctos y erróneos que podemos hacer en nuestro día a día.

Reglas:

- Empieza el jugador que, por suerte o consenso, hayan decidido los participantes.

- Cada jugador puede colocar una ficha en la mesa en cada ronda.

- La ficha que se coloque debe coincidir con la que ya esté puesta en alguno de sus lados. 
- Solo se pueden añadir las siguientes fichas en los extremos de las que ya estén dispuestas.

- En caso de que sobren fichas, si no se puede seguir jugando, se puede robar una. Después de robar, si se puede, se coloca la ficha; si no, se pasa el turno. Cuando no se pueda robar, simplemente se pasa el turno.

- Pueden jugar de 2 a 4 participantes.

- Gana el primer jugador que se quede sin fichas.

\section{Parejas saludables:}

Es un juego de cartas donde se tienen que buscar las parejas dando la vuelta a las cartas. Se colocan todas las cartas encima de la mesa en modo de cuadrícula, es decir, se hacen cinco filas de diez cartas una al lado de otra; en total habrá cincuenta cartas. Los jugadores tienen que ir girando las cartas para encontrar las parejas y cuando esto ocurre, se quedan las dos cartas para hacer el recuento de cartas al final de la partida. Pero si tropezamos con una carta de un hábito no saludable se vuelven a barajar las cartas y se empieza el juego de nuevo. Al igual que el juego anterior, con la observación de las imágenes el alumnado podrá reconocer lo que es un hábito saludable y lo que no.

Reglas:

- Empieza quien saque la mayor puntuación con los dados

- Se juega en el sentido de las agujas del reloj.

- Pueden jugar de 2 a 6 jugadores.

- En cada ronda, cada jugador puede girar dos cartas para encontrar la pareja. Si la encuentra, puede seguir buscando hasta que falle, y entonces pasa el turno al siguiente participante.

- Si se gira una de las cartas trampa (las cartas con dibujos de hábitos no saludables), el jugador que la ha cogido vuelve a dejar sus parejas boca abajo y se barajan las cartas de la mesa junto con las del jugador.

- Gana el que más parejas obtenga cuando ya no queden cartas saludables en la mesa.

Para llegar a la maleta final, hicimos un prototipo de evaluación, para poder comprobar que estos materiales funcionaban. Decidimos hacer dos evaluaciones, una para otros profesionales de la educación y otra para el alumnado.

Para la evaluación docente, presentamos los juegos a compañeros de un centro de Castellón, donde creamos un debate para que ellos nos pudieran ofrecer su feedback y así poder mejorar el prototipo para la creación final. 
Después realizamos una serie de cuestionarios para el alumnado de 50 y 60 de educación primaria del mismo centro. En primer lugar, les pasamos un cuestionario de conocimientos previos sobre el contenido de actividad física y salud. Otro día, practicamos uno de los juegos con toda la clase, por grupos cooperativos. $Y$ finalmente, otro día distinto, volvimos a pasar el cuestionario para observar si habían mejorado sus conocimientos. Al acabar, les preguntamos que les había parecido el juego, si les había gustado y si las reglas eran claras, si modificarían algo, etc. De este modo, recogimos también la opinión de los discentes, muy importante para que un recurso funcione y sea motivante.

\section{Resultados y conclusiones}

En primer lugar, con la evaluación de los docentes, se realizó un debate donde adquirimos datos cualitativos para mejorar nuestro recurso. Consistió en un debate en el que nos indicaron algunas estrategias para que los juegos fueran más motivadores y atractivos para el alumnado, pero no modificaban el contenido ni la dinámica de los juegos.

En segundo lugar, en lo que se refiere a la evaluación del alumnado, a continuación podemos observar gráficamente los resultados objetivos obtenidos en la evaluación cuantitativa de los cuestionarios, tanto en el aula de 5. ㅇ B como en 6.ㅇ A:

En segundo lugar, en lo que se refiere a la evaluación del alumnado, a continuación podemos observar gráficamente los resultados objetivos obtenidos en la evaluación cuantitativa de los cuestionarios, tanto en el aula de 5. o B como en $6 .$. A:

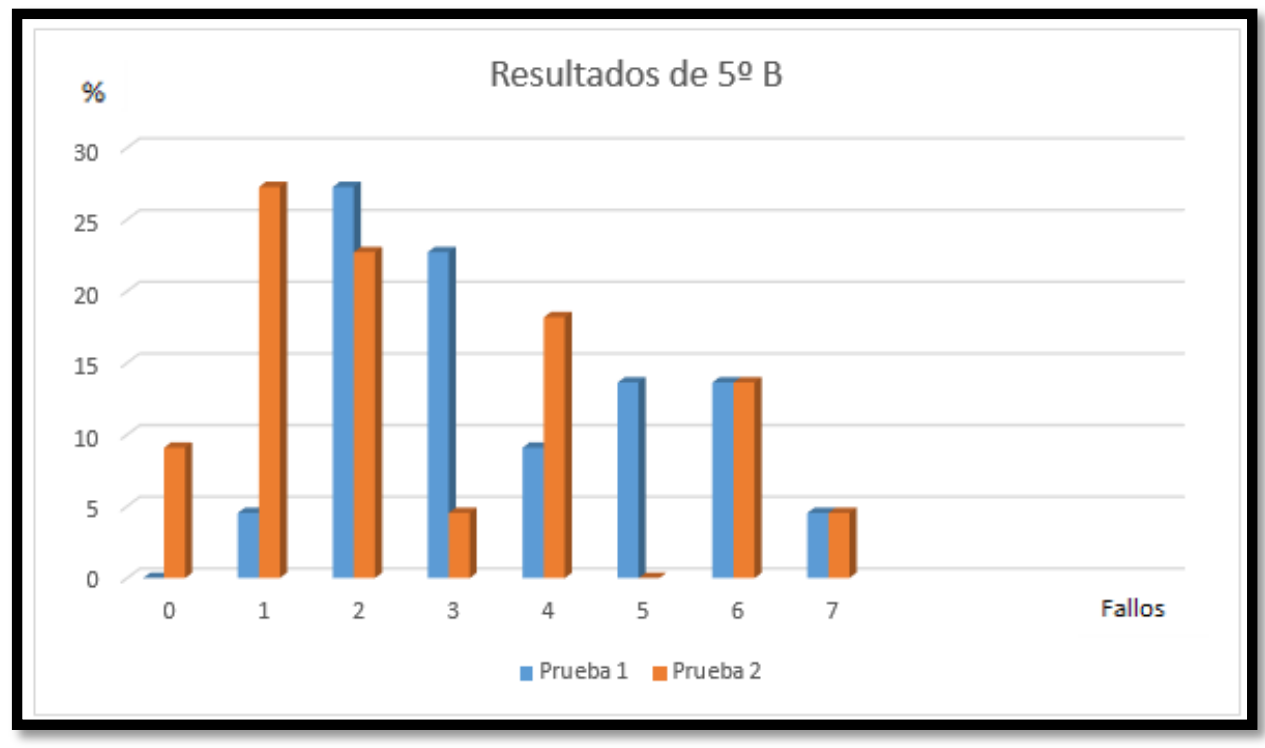

Figura 2. Resultados de las pruebas de 5.ㅇ B 


\section{$\mathbf{1}^{\text {er }}$ cuestionario de $5^{\circ}$ curso, 21 alumnos: \\ $2^{\circ}$ cuestionario de $\mathbf{5}^{\circ}$ curso, 21 alumnos: \\ 3,6 fallos de media, $17 \%$

$$
\text { - 2,6 fallos de media, } 12 \%=\downarrow \mathbf{5} \%
$$

Figura 3. Media aritmética de los resultados

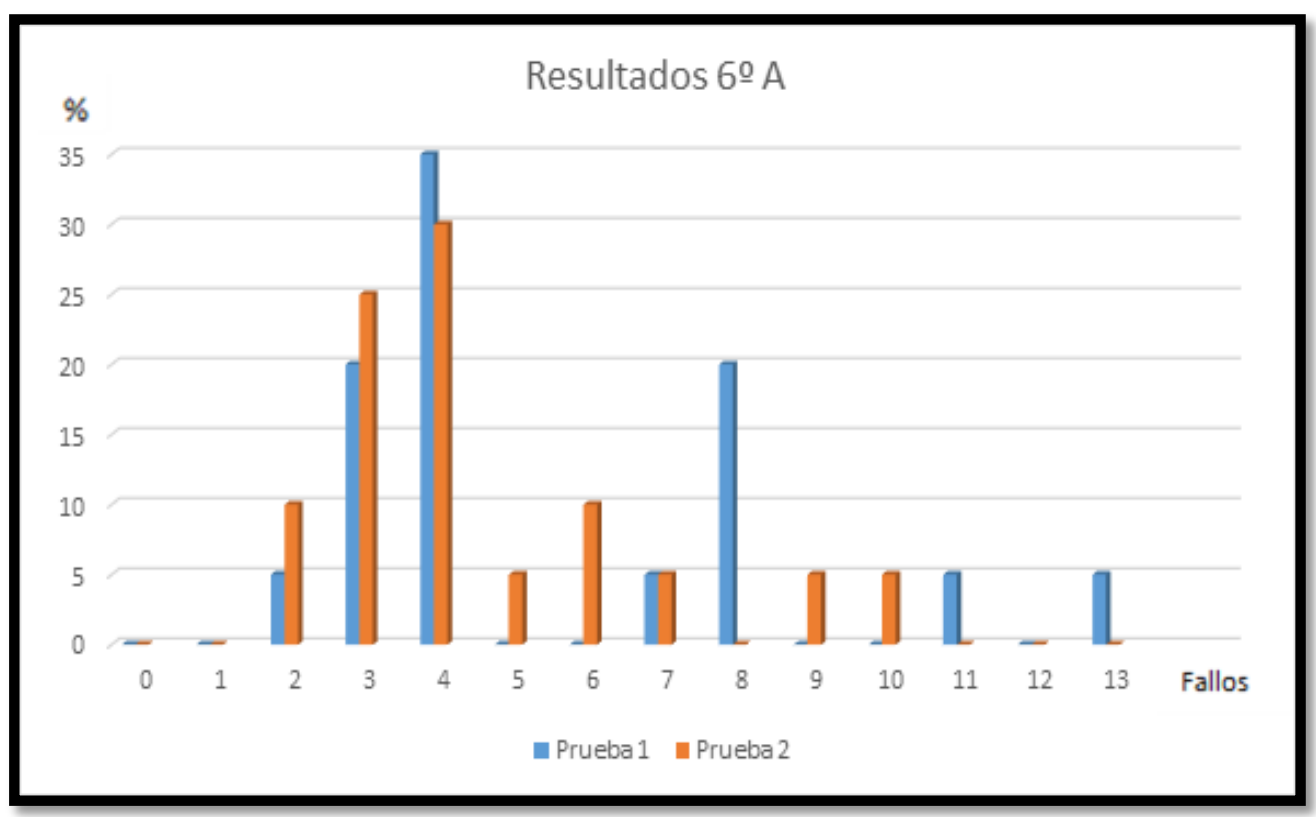

Figura 4. Resultados de las pruebas de 6.ํA

\begin{tabular}{|l|l}
\hline $1^{\text {er }}$ cuestionario de $\mathbf{6}^{\circ}$ curso, $\mathbf{1 9}$ alumnos: & $2^{\circ}$ cuestionario de $\mathbf{6}^{\circ}$ curso, $\mathbf{1 9}$ alumnos: \\
5,5 fallos de media, 29\% & -4 fallos de media, $21 \%=\mathbf{1} \% \%$
\end{tabular}

Figura 5. Media aritmética de los resultados

Al contrastar los resultados de los datos objetivos de los dos cuestionarios pudimos apreciar una pequeña mejora, teniendo en cuenta que solo habíamos utilizado una sesión de 60 minutos. En 6.으 la media de fallos del primer cuestionario era de 5,5 fallos y en el segundo, de 4 fallos; así pues, se puede apreciar una disminución del $8 \%$ de fallos del primer al segundo cuestionario. Y en 5. o la media del primer cuestionario fue de 3,6 fallos y en el segundo, de 2,6 fallos. En esta ocasión, el porcentaje de disminución de errores fue del $5 \%$. Como vemos hubo menos fallos en el segundo cuestionario después de haber practicado el juego. Así pues, estos resultados de las dos evaluaciones dieron pie al producto final de nuestro trabajo.

Con respecto a la opinión del alumnado hacia el juego utilizado, nos dijeron que sí que les gustó y que no modificarían nada; que lo habían entendido todo muy bien y había sido divertido. Nuestra 
perspectiva fuera del juego fue positiva, puesto que estaban motivados y se comentaban las respuestas continuamente en un buen trabajo cooperativo.

Finalmente, el resultado final de esta investigación fue la creación de una maleta saludable, una caja reciclada que tiene un tamaño cómodo para poder trasportarla y tener el material organizado. En la tapa están las instrucciones de uso, para que cualquier docente, sin haberla visto previamente, coja la maleta y pueda utilizarla sin problemas. La maleta consta de los cinco juegos de mesa que hemos explicado anteriormente, en los que se trabaja el contenido mencionado y, además, cada juego tiene su propia ficha de instrucciones, fáciles de leer y entender tanto para el docente como para el alumnado.

A modo de reflexión final acerca de nuestra investigación, podemos asegurar que nuestro recurso didáctico favorecerá el aprendizaje de una forma lúdica y significativa. Por lo que hemos podido observar en la práctica, el alumnado se ha sentido motivado, puesto que ha sido el agente activo en su propio proceso de enseñanza-aprendizaje. Utilizando esta metodología se le ha dado autonomía $y$, por medio de la participación grupal, han trabajado cooperativamente, debatiendo las preguntas del juego antes de dar la respuesta definitiva y, sobre todo, han respetado las normas del juego y a sus iguales. Así pues, el aprendizaje se ha visto reforzado y han aprendido unos de otros. Por todo lo mencionado anteriormente, podemos confirmar que nuestro recurso didáctico realmente funciona.

Todavía cabe señalar que, como en cualquier proyecto, todo es mejorable e incluso ampliable. Por ello, pretendemos seguir trabajando y desarrollar más materiales con contenidos de EF para otras edades o añadir más actividades en la maleta.

\section{Bibliografía}

Bronfenbrenner, Urie. 2009[1979]. The Ecology of Human Development: Experiments by Nature and Design. Cambridge, Massachusetts: Harvard University Press.

Delgado, Manuel y Pablo Tercedor. 2002. Estrategias de intervención en educación para la salud desde la Educación Física. Barcelona: Inde Publicaciones.

Hernán-García, Mariano, Maria José Ramos Monserrat y Alberto Fernández Ajuria. 2001. «Revisión de los trabajos publicados sobre promoción de la salud en jóvenes españoles». Revista española de salud pública 75 (6): 491-504.

Kelder, Steven H., Chetyl L. Peny, Knut-Inge Klepp y Leslie L. Lytle. 1994. "Longitudinal Tracking of Adolescent Smoking, Physical 
Activity, and Food Choice Behaviors». American Journal of Public Health 84 (7): 1121-1126.

Ley Orgánica 8/2013, de 9 de diciembre, para la mejora de la calidad educative. BOE 295 (19 de diciembre de 2013): 97858-97921.

Mendoza, Ramón, María Reyes Sagrera Pérez y Joan Manuel Batista Foguet. 1994. Conductas de los escolares españoles Relacionadas con la salud (1986-1990). Madrid: Consejo Superior de Investigaciones Científicas.

Ministerio de Educación, Cultura, Ciencia y Tecnología (Argentina). 2006. Educar. Buenos Aires: Presidencia de la Nación. https://www.educ.ar/recursos/93110/clases-de-educacionfisica-en-dias-de-lluvia

Organización Mundial de la Salud (OMS). 1948. Constitución de la Organización Mundial de la Salud. Nueva York: Asamblea Mundial de la Salud. https://www.who.int/governance/eb/who_constitution_sp.pdf

Pazos, José María y José Luis Aragunde. 2000. Educación postural. Barcelona: INDE.

Perea, Rogelia. 1992. "Educación para la salud. Las materias transversales como criterio de calidad educativa». En III Jornadas sobre la LOGSE, 47-56. Granada: Proyecto Sur de Ediciones.

Pozuelos, Francisco José y Gabriel Travé. 1999. "Alimentación y consumo en la educación obligatoria. Una propuesta desde la didáctica globalizada». En Curso de Metodologías de información, educación y comunicación en promoción de salud. Granada: Escuela Andaluza de Salud Pública.

Real Academia Española de la Lengua (RAE). 2017. Diccionario de la Lengua Española. Madrid: RAE. http://www.rae.es

Real Decreto 1006/1991, de 14 de junio, por el que se establecen las enseñanzas mínimas correspondiente a la Educación Primaria. BOE núm. 238, de 4 de octubre de 1990.

Real Decreto 8/2013, de 9 de diciembre, por lo que se establecen los requisitos mínimos correspondientes a Educación Primaria. BOE núm. 295, de 10 de diciembre de 2013.

Saludemia.com. 2017. Saludemia. http: //www.saludemia.com/

Sánchez Bañuelos, Fernando. 1996. La actividad física orientada hacia la salud. Madrid: Biblioteca Nueva. 